\title{
Il faut soutenir la révolution scientifique africaine
}

Écrit par : Thierry Zomahoun, President and CEO, African Institute for Mathematical Sciences (AIMS) Founder and Chair, Next Einstein Forum (NEF)

Dernière mise à jour : 23 octobre 2018

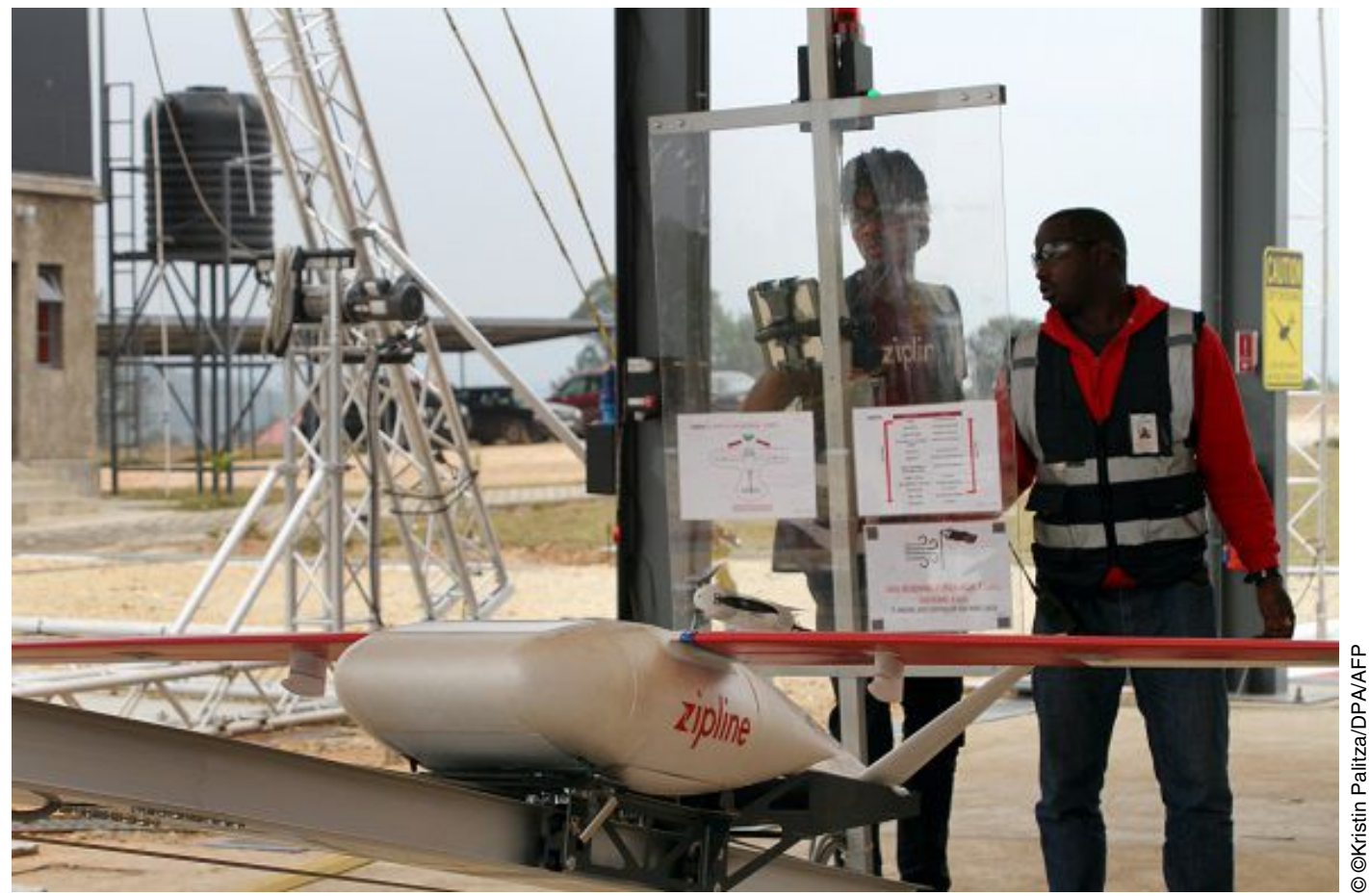

Pour accroître durablement la croissance et le bien-être, l'Afrique doit accélérer son développement par le savoir grâce à des politiques et des investissements scientifiquement étayés.

En Afrique, la base de consommateurs, les ambitions entrepreneuriales et les efforts d'innovation sont aujourd'hui en pleine croissance. Avec plus de 300 pôles technologiques répartis dans 93 des villes du continent, les créateurs d'entreprise innovent dans tous les secteurs, de l'éducation et de la santé à l'agriculture et l'énergie. Ne se contentant plus d'adapter ce qui est apparu ailleurs dans le monde, l'Afrique devient elle-même productrice de connaissances, d'innovation, de créativité et de technologies. Mais il ne fait aucun doute que l'action publique africaine pourrait accélérer ce processus. Le modèle de développement par le savoir, qui fait ici encore figure de nouveauté, devrait définir nos objectifs collectifs. L'émergence d'une population africaine jeune, éduquée, compétente et créative, parallèlement à la mise en place de politiques et d'investissements 
publics nationaux et panafricains scientifiquement étayés, pourrait largement transformer la société et améliorer le bien-être.

L'application en Afrique d'un modèle de développement par le savoir s'est accélérée du fait de l'arrivée de créateurs de connaissances et d'innovateurs occidentaux et asiatiques attirés par la flexibilité du cadre réglementaire du continent. L'Afrique a ainsi pu accueillir les premiers déploiements de technologies nouvelles, l'un des exemples notables à cet égard étant celui de drones de livraison de produits médicaux, une solution transversale destinée à pallier les défaillances de l'infrastructure médicale du continent.

Il en ressort que le développement par le savoir et par la science est indispensable à l'équation du succès suivante :

Innovation à création d'emploi à inclusion socio-économique à progrès social à gains pour l'ensemble de la population d'un pays

Pour résoudre cette équation, les pouvoirs publics africains doivent d'abord prendre des mesures concrètes et spécifiques afin de favoriser la production et la diffusion des connaissances sur le continent. Des stratégies devraient être délibérément centrées sur trois grandes questions : (1) comment améliorer les cadres de réglementation nationaux pour mettre en place des sociétés du savoir, en particulier dans les deux domaines stratégiques de l'industrie et de la science ;

(2) comment encourager les compétences et les capacités adaptées à l'implantation d'une culture de la science et de la créativité en Afrique ; (3) comment concevoir des partenariats efficaces et des mécanismes de financement structurés pour contribuer à la réalisation des deux objectifs précédents.

Prenons l'exemple del'économie circulaire sobre en carbone. Grâce au rythme de croissance du secteur industriel, l'Afrique pourrait être en mesure de sauter certaines étapes de l'économie linéaire classique pour s'engager directement dans des modèles circulaires, écologiquement plus responsables, et récolter les bénéfices de l'intégration des facteurs sociaux, économiques et environnementaux. En nous intéressant à l'empreinte environnementale des procédés industriels à l'échelle du cycle de vie, nous pouvons non seulement réduire leurs impacts négatifs, mais aussi identifier de nouvelles opportunités d'innovation et de création de richesse. Pour y parvenir, il est possible d'utiliser des matières et des énergies renouvelables, de concevoir et de fabriquer des produits à faible impact réparables et améliorables, ou de recycler les matières premières.

Pour assurer une transition rapide de l'Afrique vers l'économie circulaire, les pouvoirs publics, les universités, le secteur privé et la société civile doivent œuvrer ensemble à la création d'un environnement propice à la recherche et au développement, orienté vers une économie bas carbone à faible impact. Les moyens d'y parvenir incluent notamment l'utilisation de matières et de ressources énergétiques biosourcées plutôt que dérivées du pétrole ; le 
développement de produits composés de ressources renouvelables qui réduisent autant que possible la consommation d'énergie ; la production de biens à l'échelle locale, en utilisant des ressources renouvelables locales ; le soutien aux efforts de récupération des déchets visant à en faire les ressources de nouveaux procédés industriels, etc. En substance, il s'agit de donner la priorité aux ressources renouvelables qui nous entourent, et d'éviter qu'elles ne deviennent des déchets.

De plus, les parties prenantes africaines doivent privilégier l'indépendance énergétique. La catastrophe nucléaire de Fukushima Daiichi a marqué un tournant dans la transition énergétique européenne vers un déploiement rapide des énergies renouvelables, qui sont particulièrement abondantes en Afrique. Le tissu économique et social africain pourrait s'en voir transformé dans les années à venir. Néanmoins, pour assurer à la fois un accès universel à l'énergie et l'indépendance énergétique, il nous faudra surmonter des obstacles majeurs. Malgré les progrès rapides des dispositifs de captage, de stockage et de gestion des sources renouvelables, des investissements colossaux sont nécessaires pour financer des infrastructures et des systèmes financièrement viables et techniquement réalisables. Ceci nécessitera un soutien politique fort, avec des incitations et des réglementations à l'appui des investissements publics et privés, ainsi que des partenariats pour réaliser des avancées technologiques. Ce sera le cas notamment dans le domaine du stockage de l'énergie, pour trouver des solutions à l'intermittence des énergies renouvelables.

En Afrique, l'option privilégiée de stockage des énergies renouvelables devrait être la production de combustibles de synthèse sous forme gazeuse ou liquide, par opposition aux batteries. Dans le cadre des tables rondes de notre Next Einstein Forum, nous continuons d'étudier d'autres voies d'innovation au service de la prospérité. Très impliqué dans le domaine des politiques scientifiques et industrielles, l'Institut africain des sciences mathématiques (AIMS) est également actif dans de nombreuses disciplines d'enseignement et de recherche, notamment le changement climatique, les mathématiques pures et appliquées à la finance, les données massives et l'intelligence artificielle.

L'AIMS est convaincu qu'une culture de l'innovation ouverte, construite sur des partenariats le long de la chaîne de valeur de l'éducation, est fondamentale pour commercialiser le socle croissant de connaissances de l'Afrique et pour monter en gamme dans la chaîne de valeur mondiale. C'est à ce niveau que la coopération internationale pourrait avoir un impact à long terme.

@L'Observateur de l'OCDE, novembre 2018

\section{Références}

www.visualcapitalist.com/africa-exploding-tech-startup-ecosystem www.visualcapitalist.com/africa-exploding-tech-startup-ecosystem

www.nef.org www.nef.org 